\title{
Risk Assessment of Progressive Casino Games
}

John Quigley

University of Strathclyde, Glasgow, UK

Matthew Revie*

University of Strathclyde, Glasgow, UK

* Corresponding author. Address for correspondence

Department of Management Science, University of Strathclyde, 40 George St, Glasgow, G1 1QE, $U K$.

\footnotetext{
Abstract

This paper presents an investigation into the properties of a stochastic process whereby the value of a fund grows arithmetically and decays geometrically over discrete time periods. While this general structure is applicable to many situations, it is particularly prevalent in many casino games. This investigation was motivated by a request for support by a casino operator. Statistical models were developed to identify optimal decisions relating to the casino game concerning setting the initial jackpot, the probability of winning each prize, and the size of the prizes. It is demonstrated that all moments of the process converge asymptotically and the limiting distribution is not Normal. Closed form expressions are provided for the first moment as well as investigate the quality of approximating the distribution with an Edgeworth Expansion. The case that motivated this initial investigation is presented and discussed.

Keywords: Stochastic Processes, Gaming, Risk, Optimisation
} 


\section{$1 \quad$ Introduction}

There were 144 casinos operating in the UK with a workforce of 14,667 in 2012 (Gambling Commission, 2010) with an estimated 5,000 casinos worldwide. In the UK, almost $f 6$ Billion was gambled in 2012 (Gambling Commission, 2010), while worldwide, approximately \$100 Billion was gambled in casinos. The casino industry is becoming more substantial with worldwide growth expected to be around 9\%; fuelled in part by large growth in Asia (PriceWaterhouseCooper, 2010). In the largest markets, for example the US, China, Singapore, UK and Australia, gambling is heavily regulated by the government. Regulation is used to ensure that games are accurately advertised to players and that minimum payouts are made. This paper focuses on regulation within the UK only; however, similar regulation exists in all the major markets. A key part of the regulation is ensuring that the risk associated with a game is well understood. However, as games become more complex, intuition is challenged and there is a greater need for stochastic models to explicate the relationship between setting the conditions of the games and outcomes.

Decision support models such as optimization, stochastic modelling, data mining, decision theory and forecasting, have played a key role in supporting decision making within casinos. For example, optimization was used by Bayus and Gupta (1985) to model floor configurations of slot machines, data mining was used by (Hendler and Hendler, 2004) to model revenue management of a casino, prospect theory was used by (Barberis, 2012) to model player strategy, while mathematical models have been used to model casino VIP rebate schemes (Gao et al., 2011). (Gainsbury et al., 2012) analysed player data to assess the behaviour of different player types. More broadly, optimisation was used by (Mills and Panton, 1992) to optimizing security officers, decision theory by (Tsoukias, 2008) to model rationality of players, and data mining by (Faregh and Leth-Steensen, 2011)to model game preferences of players. Statistics has also been used to support player decision making. Werthamer (Werthamer, 2005) modelled optimal betting schemes for players on blackjack, and then extended this work in (Werthamer, 2008) to model players 'hopping' between tables to maximize expected return. As illustrated, decision support models have played a key role in supporting decision making across a wide range of casino operations, including the development and design of casino games.

In this paper, we are concerned with supporting a casino set the operational parameters of a new game that is being implemented across a chain of casinos. This analysis takes two forms. 
First, we model the stochastic behaviour of the game. Second, we optimize the profitability of the game through modification of these initial operational parameters and by capturing the arrival rate of players through a function of the jackpot.

The game is structured as follows. The value of the jackpot following the $n^{\text {th }}$ hand is denoted by $F_{n}$. We refer to each play as a hand and a sequence of hands terminating in the jackpot being won is referred to as a game. Such games start with an initial amount of money in the jackpot provided by the casino which we denote by $F_{0}$. Setting the value of $F_{0}$ is one of the key decisions that the casino operators must make for the game. Hands are then played sequentially. Each player pays $£ 1$ to play, of which the casino takes $f h$ and the remaining $f$ $1-h$ is added to the jackpot. The casino can set the value of $h$ but the regulator bounds it between 0 and 0.3. A random mechanism then determines the winnings to the player, which comprise a) nothing, b) a fixed amount, or c) a proportion of the jackpot, which may include winning the entire jackpot. The probabilities associated with the prizes do not change throughout the game, but can be determined by the casino at the beginning. The winnings are removed from the jackpot and if the value of the jackpot is greater than 0 , then the game continues.

Through deriving an explicit expression of the value of the jackpot (similar to the work of (Sugden, 2010) applied to Keno) we support decisions made by the Director of Operations for the casino in determining appropriate probabilities and associated prizes. The decisions that we support include the following: the size of the initial jackpot; the size of the contribution to the jackpot per hand; the proportion of the jackpot won; the size of each prize; and the probabilities associated with each prize. In addition, our analysis reveals behaviour of the game that is counterintuitive, demonstrating the need for analytical investigation of the process.

For our initial study, we were interested in a particular game structure described by the casino. However, this general description of progressive jackpots is applicable to various casino games. Progressive side wagers (Nevada Gaming Commission and State Gaming Control Board, 2005), are games with a progressive jackpot available to players playing a traditional game, where the random mechanism that determines the prize is derived from the realizations on-going during the traditional game. For example, during a game of blackjack, players will be provided the opportunity to make additional bets on the outcome of the cards dealt through playing a 
progressive jackpot, where prizes will be allocated depending on the cards realized. The structure of the game remains identical so that players can play both the traditional game and the progressive game.

The structure of the paper is as follows. In Section 2, closed form solutions for the mean of the value of the fund are presented. The asymptotic behaviour of the first moment is assessed. In Section 3, an Edgeworth expansion is derived for approximating the distribution function of the value of the jackpot. The case that motivated this investigation, based on analysis commissioned by a UK casino chain, is presented in Section 4. By modelling the arrival rate of customers as a function of the jackpot, we optimize the initial conditions of the game to maximize profit over calendar time. Finally, in Section 5 we summaries and conclude the paper, and outline future work.

\section{$2 \quad$ Deriving Moments}

\section{$2.1 \quad$ Outline}

In this section we derive an explicit expression for the first moment associated with progressive jackpot games. In Lemma 1 we set up a recursive expression for deriving all moments. This is used to determine an explicit expression for the first moment described in Theorem 1 . This is followed by Corollary 1 which establishes an asymptotic expression for the first moment of the jackpot, showing it converges to a finite mean. Corollary 2 establishes that this convergence is monotonic. Theorem 2 derives conditions for all moments of the series to converge to a finite expression. Theorem 3 presents an expression for the Moment Generating Function of the series, followed by Corollary 3 , which presents a general expression for all asymptotic moments and can be used to show that the limiting distribution of the value of the jackpot is not Normal. All proofs are in the Appendix.

The results from this section will be used in Section 3 for deriving an Edgeworth Expansion to approximate the distribution function of value of the jackpot. The analysis and derivations in Section 2 and Section 3 are conditional on the jackpot not being won. 
Throughout this section and the remainder of the paper, the notation described in Table 1 will be used.

\begin{tabular}{|c|c|}
\hline Symbol & Description \\
\hline$F_{0}$ & Initial fund provided by the casino \\
\hline$p$ & Probability jackpot is won \\
\hline$F_{i}$ & $\begin{array}{l}\text { Random variable representing the value of the fund after } i \text { hands have } \\
\text { been played }\end{array}$ \\
\hline$q$ & Probability of winning proportion of jackpot \\
\hline$\gamma$ & Proportion of jackpot won as proportional prize \\
\hline$I_{i}$ & $\begin{array}{l}\text { Random variable indicating whether the proportional win has been made } \\
\text { on the } i \text { th hand }\end{array}$ \\
\hline$X$ & $\begin{array}{l}\text { Random variable representing fixed amount win given the proportional } \\
\text { prize is not won where each } X_{N} \text { has the same distribution as } X\end{array}$ \\
\hline$J_{i}$ & $\begin{array}{l}\text { Random variable indicating whether or not the jackpot has been won on } \\
\text { the } i \text { th hand }\end{array}$ \\
\hline$h$ & Value taken by the casino on each hand \\
\hline
\end{tabular}

Table 1: List of notation used throughout the paper

\section{$2.2 \quad$ Closed form solution of first moment}

We consider the progressive jackpot with one of two types of prizes. A fixed prize is awarded on the $n^{\text {th }}$ hand with a specified probability that will result in an arithmetic change on the series denoted by $X_{n}$. A proportion $\gamma$ of the jackpot is awarded that will result in a geometric change in the value of the jackpot. We can express the value of the jackpot following the $n^{\text {th }}$ hand recursively in the following manner through a conditioning argument, where the indicator 
variable $I_{\gamma}$ denotes whether the proportional prize has been won. As such the random variable $X_{n}$ is conditional on the proportional prize not having been won:

$$
\begin{gathered}
F_{n} \quad=\left(F_{n-1}+(1-h)-X_{n}\right)\left(1-I_{\gamma}\right) \\
+\left(F_{n-1}+(1-h)\right)(1-\gamma) I_{\gamma}, n=1,2, \ldots
\end{gathered}
$$

where: $I_{\gamma}= \begin{cases}1, & \text { if proportional prize awarded; } \\ 0, & \text { else }\end{cases}$

The following key assumptions are made:

1. The initial jackpot is $F_{0}$.

2. Each $X_{n}$ is independently and identically distributed for all $n$.

3. $1-h$ is added to the jackpot for each hand as it costs 1 unit to play and the casino take $h$.

4. A proportion $\gamma$ of $F_{n}$ can be won on the $n^{\text {th }}$ hand with probability $q$.

Our first result presents a recursive relationship, permitting the evaluation of all moments for the jackpot at a specified hand $n$.

\section{Lemma 1}

$$
E\left[F_{n}^{j}\right]=\sum_{i=0}^{j} w_{i j} E\left[F_{n-1}^{i}\right]
$$

Where $w_{i j}=\left(\begin{array}{c}j \\ i\end{array}\right)\left[E\left[(1-h-X)^{j-i}\right](1-q)+(1-h)^{j-i}(1-\gamma)^{j} q\right]$ 
The Lemma can be used to derive closed form expression for moments. Upon inspection of the weights, we see that moments can be expressed as sums of geometric series. Theorem 1 makes use of this relationship and provides a closed form expression for the first moment of the jackpot for any specified hand $n$.

\section{Theorem 1}

Given that the four assumptions hold, then the expectation of the jackpot after $n$ hands is:

$E\left[F_{n}\right]=(1-\gamma q)^{n} F_{0}+((1-h)(1-\gamma) q+((1-h)-E[X])(1-q)) \frac{1-(1-\gamma q)^{n}}{\gamma q}$

Depending on the game parameters, the expectation of the jackpot $F_{n}$ decreases monotonically, increases monotonically or remains constant with respect to the number of hands $n$. This is of interest in determining the conditions of the game, such as the probability of winning, the associated prizes and the initial value of the jackpot, as it would be undesirable for a casino to have an expected jackpot that decreases over time.

From this, we can develop two corollaries. These two corollaries are not immediately intuitive but they naturally emerge from Theorem 1 . Corollary 1 demonstrates that the jackpot reaches a limit; which is not in itself immediately obvious.

\section{Corollary 1}

The limit of $E\left[F_{n}\right]$ as $n \rightarrow \infty$ is $\lim _{n \rightarrow \infty} E\left[F_{n}\right]=\frac{(1-h)(1-\gamma) q+E[1-h-X](1-q)}{\gamma q}$ 
Upon inspection of Theorem 1 we can observe that the series will converge to a finite mean under certain conditions. The explanation as to why the fund converges is because the proportional prize, i.e. \$gamma\$, discounts the influence of the past geometrically. Corollary 2 provides conditions as to when it is increasing, decreasing or stationary.

\section{Corollary 2}

$$
\begin{aligned}
& \Delta E\left[F_{n}\right]>0 \text { if } \frac{((1-h)(1-\gamma) q+((1-h)-E[X])(1-q))}{\gamma q}>F_{0}, \\
& \Delta E\left[F_{n}\right]<0 \text { if } \frac{((1-h)(1-\gamma) q+((1-h)-E[X])(1-q))}{\gamma q}<F_{0}, \\
& \Delta E\left[F_{n}\right]=0 \text { if } \frac{((1-h)(1-\gamma) q+((1-h)-E[X])(1-q))}{\gamma q}=F_{0} .
\end{aligned}
$$

The impact of Corollary 2 is that the fund mean may be monotonically decreasing. The Director of Operations stated that this was something the casino would wish to avoid.

\subsection{Asymptotic Expressions for Moments}

We can extend the results from Lemma 1 to derive a recursive asymptotic expression for all moments of the series. This is stated in Theorem 2. In combination with Corollary 2, these are easily derived.

\section{Theorem 2}




$$
\begin{gathered}
E\left[F_{\infty}^{j}\right] \quad=\frac{\sum_{i=0}^{j-1} w_{i j} E\left[F_{\infty}^{i}\right]}{1-w_{j j}} \\
\text { where: } E\left[F_{\infty}^{i}\right] \quad=\lim _{n \rightarrow \infty} E\left[F_{n}^{i}\right]
\end{gathered}
$$

Finally, we derive an asymptotic expression for the Moment Generating Function in Theorem 3. We make use of the Moment Generating Function in Corollary 3 to obtain an expression for the asymptotic cumulants. These are used in Section 3 with the Edgeworth Expansion.

\section{Theorem 3}

The Moment Generating Function of the value of the jackpot after the $n^{\text {th }}$ hand assuming it has not been won, i.e. $M_{F_{n}}(z)$, is:

$$
\begin{gathered}
M_{F_{n}}(z)=e^{z(1-h)(1-\gamma)} M_{F_{n-1}}(z(1-\gamma)) q+M_{F_{n-1}}(z) M_{(1-h)-X}(z)(1-q) \\
\text { where } M_{(1-h-X)}(z)=E\left[e^{z(1-h-X)}\right] .
\end{gathered}
$$

\section{Corollary 3}

We denote the limit of the moment generating function as $\lim _{n \rightarrow \infty} M_{F_{n}}(Z) \equiv M_{F_{\infty}}(Z)$. From this,

$M_{F_{\infty}}(z)=\frac{e^{z(1-h)(1-\gamma)} q}{1-M_{(1-h)-X}(z)(1-q)} M_{F_{\infty}}(z(1-\gamma))$ 
It can be shown through comparing coefficients from a series expansion in $\$ z \$$ that the Moment Generating Function of the Normal distribution cannot be represented in the form expressed in Corollary 3. As such, the limiting distribution of $F_{n}$ is not Normal.

Using the moment generating function, we can develop the cumulant generating function. The relationship between moments and cumulants can be expressed recursively through the following relationship (Small, 2010):

$$
E\left[F_{n}^{i}\right]-\kappa_{i, F_{n}}=\sum_{j=1}^{i-1}\left(\begin{array}{c}
i-1 \\
j-1
\end{array}\right) \kappa_{j, F_{n}} E\left[F_{n}^{i-j}\right] .
$$

From this, the following corollary emerges.

\section{Corollary 4}

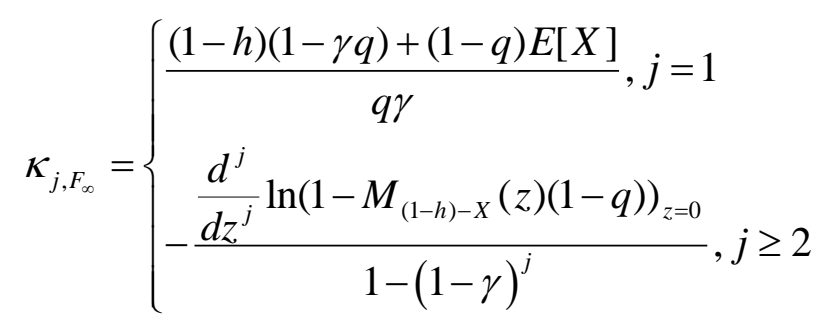

\section{$2.4 \quad$ Summary}

We can see upon inspection that the moment generating function expressed in Corollary 3 does not possess the form of the moment generating function of a Normal distribution and as such the limiting distribution of the value of the jackpot does not converge to a Normal distribution. However, we have not established how close an approximation the Normal distribution will be to the actual asymptotic distribution, which is considered in the following section. In Section 3, we illustrate that the Edgeworth Expansion will converge as a limiting series to the asymptotic distribution, and as such provide a good approximation through adjusting the Normal 
distribution for skewness and kurtosis. In Section 3, we derive an Edgeworth Expansion to describe the probability density function (PDF) as well as the Cumulative Distribution Function (CDF) of the value of the series. This is evaluated and benchmarked against the Normal distribution, where we see the Edgeworth Expansion substantially outperforms the Normal approximation.

\section{$3 \quad$ Edgeworth Expansion}

\subsection{Outline}

The Central Limit Theorem states that under certain conditions, a suitably re-scaled average of a sample of random variables will converge to the standard Normal distribution as the sample size increases. The Edgeworth Expansion was derived to improve upon the Normal approximation of the distribution of such an average for finite sample sizes. The adjustments that are proposed incorporate information pertaining to the higher moments or cumulants of the distributions. In theory, the expansion can be extended to incorporate any number of higher moments but most common are to include information about the third and fourth moment to assess skewness and kurtosis. (Hall, 1997) credits (Chebyshev, 1890), (Edgeworth, 1896) and (Edgeworth, 1907) with the initial conception of the idea.

The Edgeworth Expansion is an infinite series expansion starting after a Normal approximation to adjust for skewness, kurtosis and higher moments or cumulants. To ensure convergence we require $E\left[e^{F_{n}^{2}}\right]<\infty$ ((Cramer, 1928) as cited in (Hall, 1997). In this case, for each given $n, F_{n}$ can only take a finitely many real values; hence the expectations are less than infinity.

We propose using the Edgeworth Expansion for approximating the distribution of $F_{n}$ for large values of $n$. We know from Section 2 that the moments converge and the convergent distribution is not Normally distributed. We seek to obtain a suitable approximation of the asymptotic value of jackpot to explicate the relationship between the structure of the game and value of the jackpot. 
In Section 3.2 we show an expression of the CDF for $F_{n}$ from the Edgeworth Expansion. The Edgeworth Expansion was conceived to support inference on averages, but we propose using it on a sample of 1 in the sense that it is the realization of one sample path of the stochastic process. As such, we seek to evaluate the error associated with the approximation. This is considered in Section 3.3.

\subsection{Derivation of the Edgeworth Expansion}

Initially we re-scale $F_{n}$ such that it has a mean of 0 and a standard deviation of 1 . This is expressed as $W_{n}$ in the following: $W_{n}=\frac{F_{n}-\kappa_{1, F_{n}}}{\sqrt{\kappa_{2, F_{n}}}}$.

For the remainder of this paper, we will consider an Edgeworth approximation of the distribution of $F_{n}$ by truncating the Edgeworth expansion after the fourth moment. This truncated Edgeworth expansion is expressed in (6), see (Small, 2010).

$$
\begin{aligned}
P\left(W_{n}<w\right)= & \Phi(w)-\frac{\kappa_{3, F_{n}}}{6 \kappa_{2, F_{n}}^{\frac{3}{2}}}\left(w^{2}-1\right) \phi(w) \\
& -w\left(\frac{\kappa_{4, F_{n}}}{24 \kappa_{2, F_{n}}^{2}}\left(w^{2}-3\right)+\frac{\kappa_{3, F_{n}}^{2}}{72 \kappa_{2, F_{n}}^{3}}\left(w^{4}-10 w^{2}+15\right)\right) \phi(w)
\end{aligned}
$$

where $\Phi(w)$ and $\phi(w)$ are the CDF and PDF of the standard Normal distribution respectively.

Inspecting the expression of the CDF in equation ( $\backslash$ ref $\{$ eq_cdf $\}$ ), we see that the PDF of the Normal distribution acts as a weighting function, where the weight will be greatest near the mean. Moreover, the third cumulant $\kappa_{3, F_{N}}$ measures the effect of skewness, either increasing or decreasing the percentile obtained from the Normal distribution. 


\subsection{Assessing the accuracy of the Edgeworth Expansion}

In this section, we use the above expression of the Edgeworth expansion to evaluate the accuracy for a range of different parameter values. Four parameters are varied: $F_{0}$, the initial fund; $q$, the probability of winning a proportion of the jackpot; $\gamma$, the proportion of the jackpot won; and $n$, the number of hands played without the jackpot being won. We use the asymptotic estimates of the third and fourth cumulants. Table 2 illustrates the values simulated for each parameter.

\begin{tabular}{l|l}
\hline Parameter & Values taken \\
\hline$F_{0}$ & $0,1000,10000,100000$ \\
$q$ & $0.001,0.002,0.1,0.3333$ \\
$\gamma$ & $0.01,0.1,0.25,0.5,0.75$ \\
$n$ & $25000,100000,1000000$ \\
\hline
\end{tabular}

Table 2: Values assessed during simulation\}

Using Matlab R2009b, simulation was carried out to assess the size of the fund for each combination above, i.e. 240 different combinations. Each combination was simulated 10000 times. From this, the empirical CDF for the size of the jackpot given the parameter values was calculated. A comparison between the empirical distribution, and the Normal and Edgeworth expansion was carried out on four different measures. These measures were the maximum difference between the empirical and the Normal/Edgeworth expansion (denoted by $M_{N}$ and $M_{E}$ respectively), the difference at the 5 th, 50 th and 95 th percentiles (denoted by $5_{N}, 50_{N}$, and $95_{N}$ respectively for the Normal and similar notation for the Edgeworth expansion).

The results showed that on $96 \%$ of the simulations, the Edgeworth expansion outperformed the Normal distribution for the maximum error, and for the error at the 5th, 50th and 95th percentiles, outperformed the Normal distribution on $95.3 \%$ of the simulations. Figures $1 \mathrm{a}-1 \mathrm{~d}$ 
are scatterplots of the $M_{E}$ against $M_{N}, 5_{E}$ against $5_{N}, 50_{E}$ against $50_{N}$ and $95_{E}$ against $95_{N}$. As reference, they have been grouped by $\gamma$ values and the $45^{\circ}$ line is provided as a reference. Simulations where the Normal out (under) performs the Edgeworth expansion lie above (below) this line.

The Edgeworth Expansion outperforms the Normal for almost all input variables. As can be seen from Figures $1 \mathrm{a}-1 \mathrm{~d}$, the vast majority of the errors fall below the $45^{\circ}$ line, indicating that the error for the Normal distribution is larger than for the Edgeworth expansion.

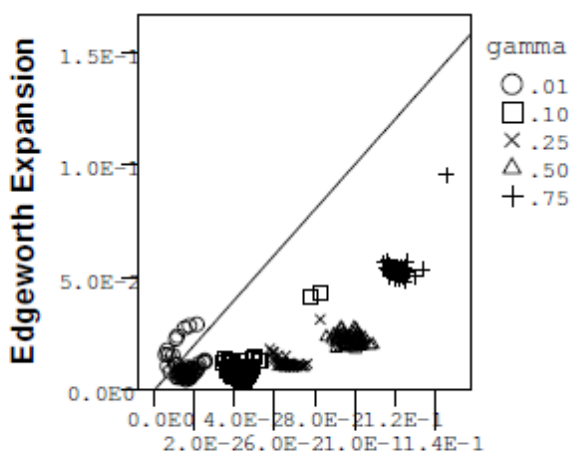

Normal Distribution

(a) Maximum error

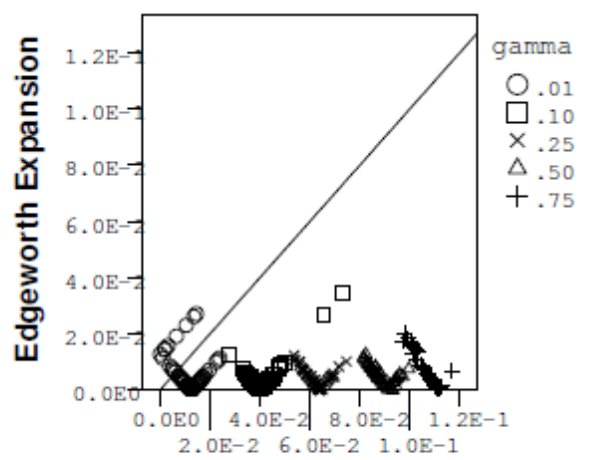

Normal Distribution

(c) Error at the 50th Percentile

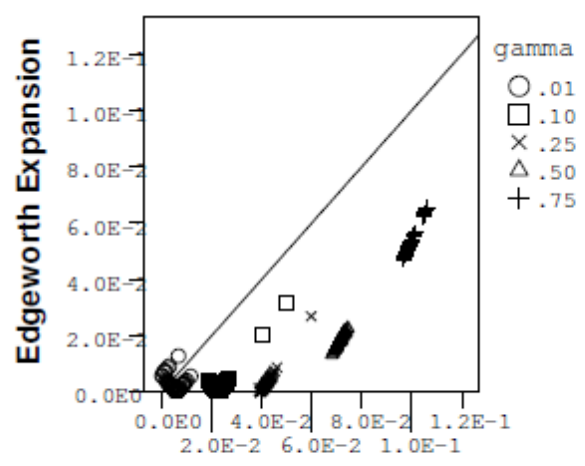

Normal Distribution

(b) Error at the 5th Percentile

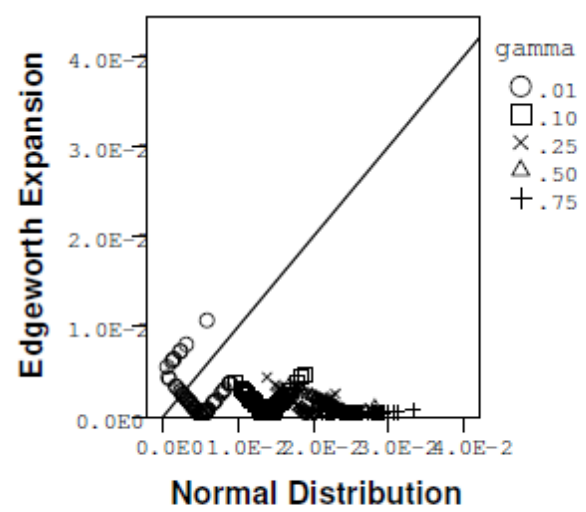

(d) Error at the 95th Percentile 
Figure 1: Scatterplot of Normal distribution against Edgeworth expansion maximum error indicating that Edgeworth expansion outperforms Normal distribution (These are labeled in the text as Figures 1a (top left), Figure $1 \mathrm{~b}$ (top right), Figure 1c (bottom left) and Figure 1d (bottom right)

Figure 1a illustrates that the maximum error for both the Normal distribution and the Edgeworth expansion appears to be randomly scattered around a central point. However, for the error assessed at percentiles, i.e. Figure $1 b-1 d$, a clear pattern emerges. As the error associated with the Normal distribution increases, for the different $\gamma$ values, the Edgeworth expansion error begins to decrease linearly, reaches zero and then increases again.

From Figures $1 \mathrm{c}$ and $1 \mathrm{~d}$, we see that the size of the error for the Normal distribution is heavily influenced by the $\gamma$ value. For each of the different performance measures, changing the value of $\gamma$ has a step change effect in the error associated with the Normal distribution. Conversely, the Edgeworth expansion, while affected, does not appear to be affected as largely by changing $\gamma$. For the 50th percentile and 95th percentile, see Figures $1 \mathrm{c}$ and $1 \mathrm{~d}$, it appears that the Edgeworth expansion error is independent of $\gamma$.

There are 25 observations (out of 300) where the Normal distribution outperforms the Edgeworth expansion for at least one of the four performance measures. Table \ref\{TB_Norm\} contains the parameter values for each of the 25 combinations. 
Table 3 Parameter values where the Normal distribution outperforms the Edgeworth expansion

\begin{tabular}{l|l|l|l||l|l|l|l}
\hline \multicolumn{1}{c|}{$F$} & $q$ & $\gamma$ & $n$ & $F$ & $q$ & $\gamma$ & $n$ \\
\hline 0 & 0.001 & 0.01 & 25000 & 1000 & 0.02 & 0.01 & 100000 \\
0 & 0.001 & 0.01 & 100000 & 1000 & 0.1 & 0.01 & 25000 \\
0 & 0.002 & 0.01 & 25000 & 1000 & 0.1 & 0.01 & 100000 \\
0 & 0.002 & 0.01 & 100000 & 10000 & 0.001 & 0.01 & 25000 \\
0 & 0.1 & 0.01 & 1000000 & 10000 & 0.001 & 0.01 & 100000 \\
0 & 0.33333 & 0.01 & 25000 & 10000 & 0.002 & 0.01 & 25000 \\
0 & 0.33333 & 0.01 & 100000 & 10000 & 0.002 & 0.01 & 100000 \\
0 & 0.33333 & 0.01 & 1000000 & 10000 & 0.3333 & 0.01 & 1000000 \\
1000 & 0.001 & 0.01 & 25000 & 100000 & 0.001 & 0.01 & 25000 \\
1000 & 0.001 & 0.01 & 100000 & 100000 & 0.001 & 0.01 & 100000 \\
1000 & 0.002 & 0.01 & 25000 & 100000 & 0.002 & 0.01 & 25000 \\
1000 & 0.002 & 0.01 & 1000000 & 100000 & 0.002 & 0.01 & 100000 \\
100000 & 0.002 & 0.01 & 1000000 & & & & \\
\hline
\end{tabular}

From Table 3, we see that $\gamma$ is the only variable that has the same value reoccurring. The only clear pattern that emerges from Table 3 is the effect of $\gamma$. For low values of $\gamma$, it appears that the Normal distribution outperforms the Edgeworth expansion - however, it is worth highlighting that the Normal distribution outperformed the Edgeworth expansion on only 25 of the 300 different combinations and on only 6 occasions, did the Normal distribution outperform the Edgeworth expansion on all four performance measures.

\section{$4 \quad$ Application}

\subsection{Outline}


In this section we illustrate the application of the results derived in Section 2 and 3 through the case which motivated the analysis. In Section 4.2 we describe the initial conditions of the game as defined by the casino. To date, no statistical techniques have been used by the casino to set these parameters. In Section 4.3 we derive expressions for the moments of the game and illustrate the implications of the conditions set by the casino as well as illustrating the Edgeworth Expansion to approximate the distribution. In Section 4.4 we explore the expected value of the jackpot when it is won given the initial parameters. In Section 4.5, we review the decisions the casino has made regarding the initial conditions and through optimization, identify optimal parameter values over calendar time.

\subsection{Initial Conditions of the Game}

The progressive jackpot under consideration for this analysis was a progressive side wager game, where additional bets would be made available during a game of blackjack. While playing a game of blackjack a player could choose to compete for the jackpot by paying $f 1$. This is in addition to the minimum wager required by the player to play the standard blackjack game. The casino take $\$ 0.3$ and contribute the remaining $\$ 0.70$ into the jackpot. The player is then dealt two cards from a shoe containing six decks. If either card is not an ace, the player loses their progressive bet. If both cards are aces, the player may continue. If the third card is not an ace, for the purposes of the progressive jackpot, they must stop; however, the player still receives the prize, i.e. once the chain is broken, they are still rewarded for their previous cards. If the third card is an ace, the player may continue. It is assumed that players will always continue to draw if they have the chance. As the probability of exceeding 21 and 'busting' at this stage is zero, this seems a reasonable assumption.

The probability of a player being dealt their first two cards as aces from the same suit is $4 \frac{P(6,2) P(288,1)}{P(312,3)}=0.0011489$. The denominator calculates the number of ways to deal 3 cards from 312, where order matters (as we must have the first two cards being aces and the last one not). The numerator calculates the number of ways the first cards can come from a particular suit and the third card not being an ace. As there are four particular suits we multiply 
by 4 . Similar calculations are conducted for each scenario. Table 4$\}$ is a summary of the different scenarios required to win, the associated probabilities and the prize. The casino start the jackpot with $\$ 10000$.

Table 4: Probability of player winning each prize

\begin{tabular}{lll}
\hline Outcome & Probability & Prize \\
\hline 2 Aces of the same suit & 0.0011489 & $£ 50$ \\
3 Aces but different suit & 0.000387767 & $£ 200$ \\
3 Aces of the same suit & 0.000015957489 & $£ 1000$ \\
4 Aces but different colour & 0.000024881 & $10 \%$ of jackpot \\
4 Aces of the same colour & 0.000002556296821 & $100 \%$ of jackpot \\
\hline
\end{tabular}

\subsection{Moments and Distribution of the Series}

We evaluate the conditional first two moments of the fixed prize awards, given that neither the proportional prize or the jackpot was won.

$$
\begin{array}{ll}
E[X] & =0.1510 \\
E\left[X^{2}\right] & =34.341
\end{array}
$$

Illustrated in Figure 2 is the expectation of the fund as a function of the number of games played. We see that the mean approaches its asymptotic value \$lunderset\{n\to linfty \}$\{\backslash$ mathop $\{\backslash \lim \}\} \backslash, E\left[\left\{\{F\} \_\{n\}\right\}\right]=£ 220,667$ assuming that no one wins the jackpot.

At its asymptotic value the expected benefit from entering the game is derived in the following way. The expected payout from playing a hand when the jackpot reaches its asymptotic value (3) is derived through combining the expected fixed payout plus the expected proportionate payout. 


$$
\begin{aligned}
E[\text { Payout }] & =E\left[X \mid I_{\gamma}=0 \cap I_{J}=0\right] \times P\left(I_{\gamma}=0 \cap I_{J}=0\right) \\
& =\lim _{n \rightarrow \infty} E\left[F_{n}\right]\left(0.1 \times P\left(I_{\gamma}=1\right)+P\left(I_{J}=1\right)\right) \\
& =£ 1.26
\end{aligned}
$$

This exceeds the fee of $£ 1$, but does illustrate that the game would require a substantial number of games to be played before the odds were in favour of the gambler.

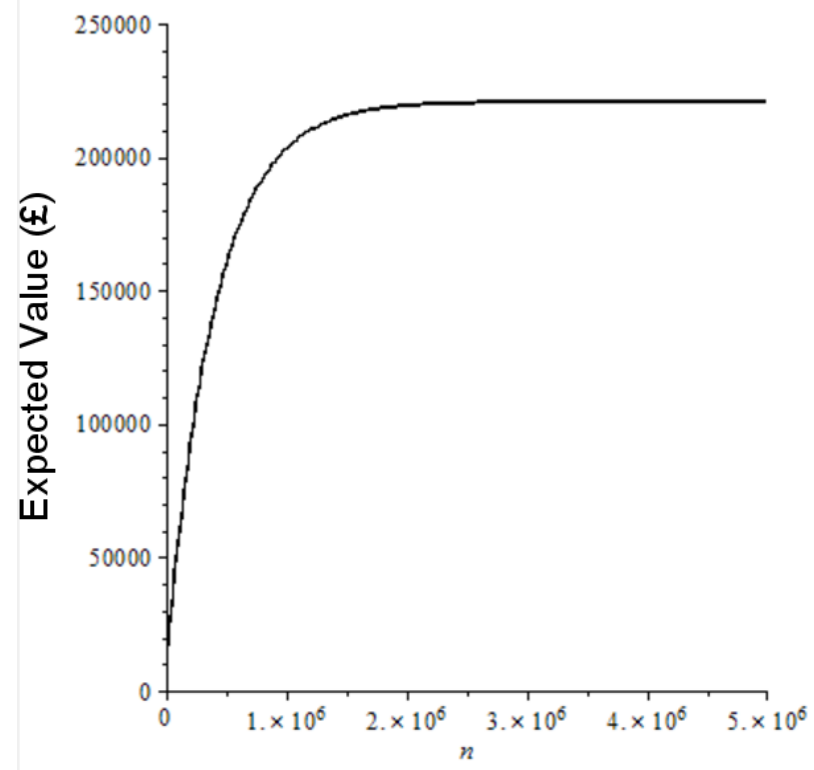

Figure 2: Expected value of the jackpot assuming that no one wins the jackpot showing the value approaching its asymptotic mean

Using Lemma 1 to determine the second moment along with the expression from Theorem 1, we can calculate the standard deviation for the series. This is illustrated in Figure 3 where we see the series approaching its asymptotic standard deviation of $£ 50,695$. 


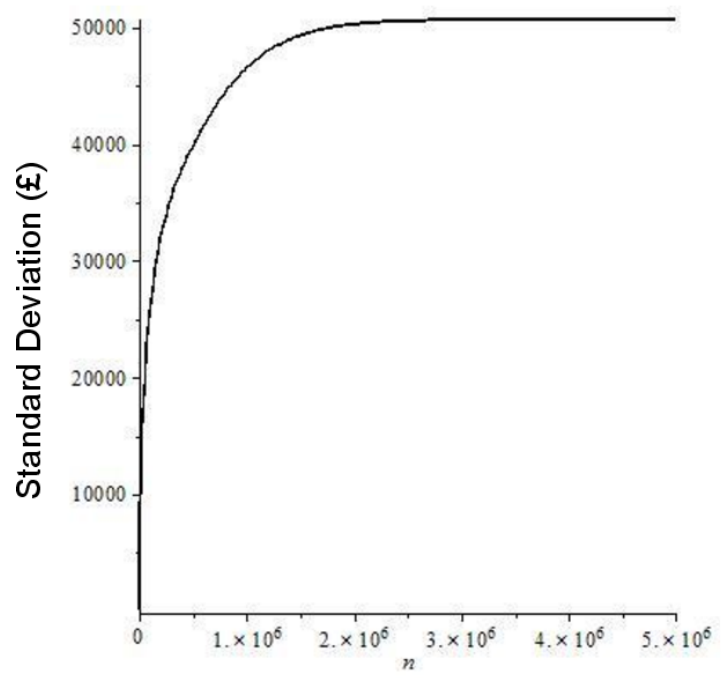

Figure 3: Standard deviation of the value of the jackpot assuming not won showing it approaching its asymptotic value\}

Finally, using Corollary 4, we can derive the asymptotic cumulants. For the Edgeworth Expansion we will require the following: $\frac{\kappa_{3, F_{\infty}}}{\kappa_{2, F_{\infty}}^{1.5}}=-0.6112$ and $\frac{\kappa_{4, F_{\infty}}}{\kappa_{2, F_{\infty}}^{2}}=0.6298$

Figure 4 illustrates the Edgeworth Expansion estimate of the asymptotic distribution of the value of the jackpot assuming it has not been won. One of the consequences of the Edgeworth Expansion is that it does not guarantee a proper PDF or CDF, such that the corrections may take the CDF above 1 or the PDF below 0. 


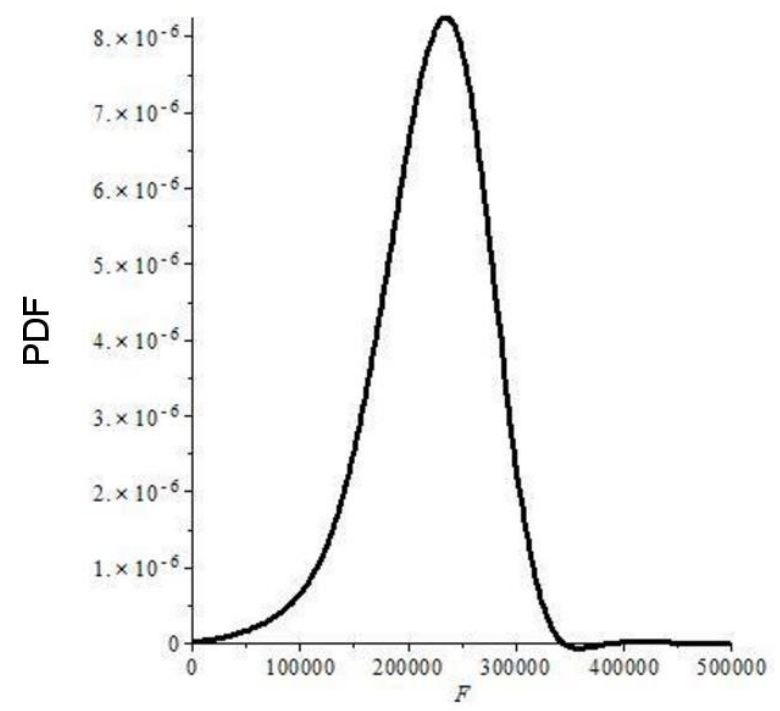

Figure 4: Edgeworth expansion for the asymptotic value of the fund

Figure 5 presents the deviation between the Normal approximation and the Edgeworth Expansion, showing the disagreement to be greatest about $f 200,000$, where the Normal approximation exceeds the Edgeworth Expansion by about 0.04. The analysis using the Edgeworth Expansion shows that the 5th and 95th percentiles are $(f 129,587, f 294,644)$. The Normal approximation would have provided a range of $(£ 137,279, £ 304,053)$. 


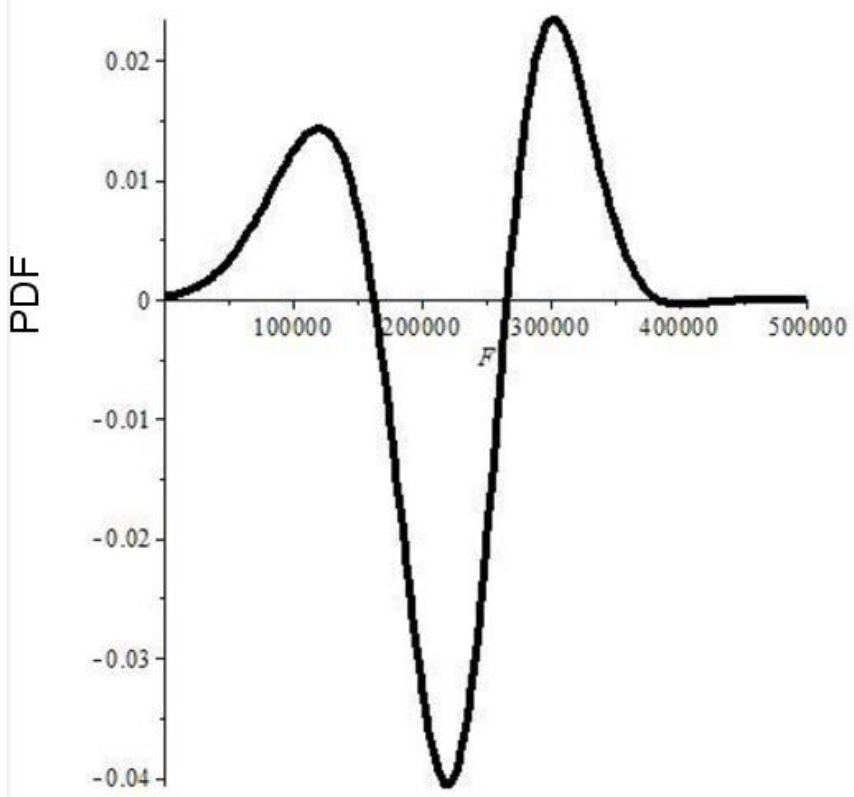

Figure 5: Difference between Edgeworth expansion and Normal Distribution showing greatest difference is almost $4 \%$

Understanding the distribution of the jackpot when won is important to the casino operations manager. Due to regulation in the UK, casinos must be able to pay out on any bet that they accept. As there may be multiple of these progressive games taking place simultaneously, and the length of time until a contribution is made to the jackpot and then when the jackpot is finally paid out, it is important that the casino appreciates the variability in the expected jackpot when it is won so they have sufficient funds available to pay the jackpot.

The analysis in this section has assumed that the jackpot has not been won. In Section 4.4 we consider the size of the jackpot when it is won.

\subsection{Expected Value of the Jackpot when it is Won}

The analysis in Section 4.2 assumed that the jackpot was not won. The random process for winning the jackpot follows a geometric distribution. As such, we can model the hand number 
used in the indexing of the jackpot value to be a random variable that follows a geometric distribution in order to estimate the value of the jackpot when it is won.

\section{Theorem 4}

Denoting the hand when the jackpot is won for the first time as $N_{J}$ and assuming the probability of winning the jackpot on any game is $p$ then the expectation of the value of the jackpot when it is won is:

$$
E\left[F_{N_{J}}\right]=\frac{p}{1-(1-p)(1-\gamma q)} F_{0}+((1-h)(1-\gamma) q+((1-h)-E[X])(1-q)) \frac{1-\frac{p}{1-(1-p)(1-\gamma q)}}{\gamma q}
$$

Using the formula provided in Theorem 4 we can calculate the expected value of the jackpot when won assuming people continue to play, which is $f 113,909$. The assumption that people continue to play is critical which we reflect on in Section 4.5 .

Lastly, the casino is interested in the expected profit and the probability of losing money within any game as they provide the initial amount into the jackpot. The expected number of hands played until the jackpot is won is simply the mean of the geometric distribution which for this series is 391,191 . As the casino earns $£ 0.7$ per hand their income per hand is expected to be $£ 273,834$. For the casino to earn back their $£ 10,000$ they require at least 14,286 hands to be played before the jackpot is won, this has a probability of 0.96 of being realized.

\section{$5 \quad$ Optimizing initial conditions}


Considering the casino as a profit maximizing organisation, it is of interest to assess the optimal initial contribution to the fund, i.e. $F_{0}$, such that profits are maximized over a period of time. So far in the analysis the stochastic process is indexed by the number of hands that have been played within any cycle. However the inter-arrival time of players will be influenced by the value of the fund and there is an obvious trade-off between having a high initial value in the fund to encourage more players, subsequently shortening the calendar length of a cycle and contributing too much so that it is unprofitable.

In this section, we consider how optimization can be used to assess the starting conditions of the game based on the arrival rate of players. We assume that the interval time between games decreases exponentially as the value in the jackpot increases. As such we can approximate the expected profit, i.e. $V$, in a given time period in the following expression: $E[V]=\left(E\left[N_{J}\right] h-F_{0}\right) e^{\lambda F_{0}}$, where $E\left[N_{J}\right]$ which is the expected number of hands per cycle, $h$ is the profit per hand, and $\lambda$ is the arrival rate of players. Here, we model that the arrival rate of the player is a function of the $F_{0}$ of the initial fund. The expected profit becomes the product between the expected profit per cycle, and the number of cycles per unit of time. Optimizing this expression with respect to $F_{0}$, to obtain the optimal value, i.e. $F_{0}^{*}$ results in the following expression: $F_{0}^{*}=E\left[N_{J}\right] h-\frac{1}{\lambda}$. This results in an expected optimal profit of $E\left[V^{*}\right]=\frac{e^{\lambda E\left[N_{J}\right] h-1}}{\lambda}$. The casino's original setting of $F_{0}$ was $£ 10000$, which would be optimal for a $\lambda$ value of approximately $\frac{1}{100000}$. Figure 6 is an illustration of the expected profits relative to the optimal value for various values of $F_{0}$ for $\lambda$ equal to $\frac{1}{1000}, \frac{1}{10000}$ and $\frac{1}{100000}$ 


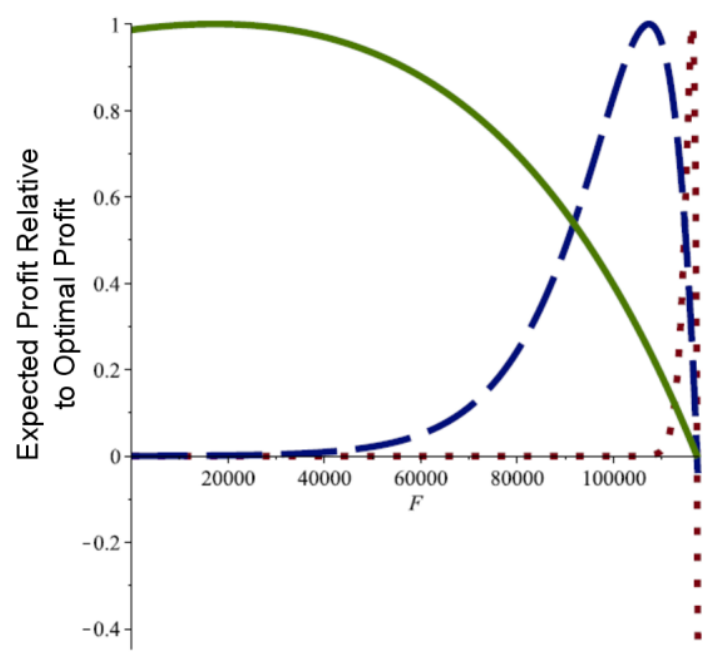

Figure 6: Illustration of the profits relative to the optimal profits with respect to $F_{0}$ for $\lambda$ values of $\frac{1}{1000}$ (dotted line), $\frac{1}{10,000}$ (dashed line) and $\frac{1}{100,000}$ (solid line) showing that profits are very sensitive for small values of $\lambda$.

Figure 6 can be used to set the initial fund of the jackpot, $F_{0}$ assuming we know the inter arrival rate of players. When the inter arrival rate is low, we should set $F_{0}$ to be small. As the inter arrival rate increases, we should increase $F_{0}$. However, prior to the game being selected, we are uncertain as to how popular the game will be. Our optimal profit for a given starting condition is very variable depending on that starting condition. For example, we can see that if we initially overestimate the number of players playing, then our expected profit is very low for $F_{0}<£ 50000$. However, if we set $F_{0} \approx 90000$, we will achieve approximately $50 \%$ of the profit for two of the scenarios.

\section{$6 \quad$ Conclusions and Future Work}


This paper has provided a modeling framework for determining the key characteristics of a casino game that can be used to inform decisions concerning optimal conditions of a game. Given the combination of prizes, from fixed amounts to proportions of jackpots with relatively small probabilities, we believe that assessing the effect on profitability is cognitively challenging. The analysis illustrates that the behavior of the process can be counterintuitive and as such requires the support of analysis. The consequences in lost opportunity costs for ad hoc decisions can be substantial with profits being very sensitive to these choices.

In the case explored, we observe that the deviation between the normal distribution and the Edgeworth expansion is relatively small (around 4\%) and as such the normal distribution provided an adequate approximation. However, in Section 3, the simulations illustrated that the corrections provided by the Edgeworth expansion could be quite substantial. Without this analysis, it would have been impossible for the Director of Operations to assess how good an approximation the Normal distribution was. Note, however, that while we have discussed the characteristics of one particular implementation of the progressive format in one game, i.e. Blackjack, the theorems developed are sufficiently generic that they could be applied to other casino games, or other situations with a similar structure.

Finally, casinos consist of games, players and spectators, and as such, are a portfolio of interacting stochastic processes that need to be managed. Through this paper, we have explicated the link between revenue and the game parameters thereby allowing decision makers to trade-off between investment, prizes and arrival rates of players. Decisions concerning the parameters of a game will influence the rate at which spectators become participants. Each casino has a finite reserve and the parameters of each games, such as $F_{0}$, must be managed simultaneously with the aim of increasing participation, not merely moving players from one game to another. From a portfolio perspective, we have not begun considering simultaneously modelling the collection of games being played within the casino. By doing so, we could optimize the parameters of each game globally against profit and risk subject to constraints such as the availability of funds to pay out winnings and initial casino reserves.

\section{Bibliography}


BARBERIS, N. 2012. A Model of Casino Gambling. Management Science, 58, 35-51.

BAYUS, B. L. \& GUPTA, S. K. 1985. ANALYZING FLOOR CONFIGURATIONS FOR CASINO SLOT MACHINES. Omega-International Journal of Management Science, 13, 561-567.

CHEBYSHEV, P. L. 1890. Sur deux theoremes relatifs aux probabilities. Acta Mathematics, 14, 305-315.

CRAMER, H. 1928. On the composition of elementary errors. Scandinavian Actuarial Journal, 1928, 13-74.

EDGEWORTH, F. 1907. On the representation of statistical frequency by a series. Journal of the Royal Statistical Society, 70, 102-106.

EDGEWORTH, F. Y. 1896. The Asymmetrical Probability Curve. Royal Society of London Proceedings Series I, 56, 271-272.

FAREGH, N. \& LETH-STEENSEN, C. 2011. The gambling profiles of Canadians young and old: game preferences and play frequencies. International Gambling Studies, 11, 23-41.

GAINSBURY, S., SADEQUE, S., MIZERSKI, D. \& BLASZCZYNSKI, A. 2012. Wagering in Australia: A retrospective behavioural analysis of betting patterns based on player account data. The Journal of Gambling Business and Economics, 6, 50-68.

GAMBLING COMMISSION 2010. Gambling Commission - Industry Statistics 2009/2010.

GAO, J. Z., FONG, D. \& LIU, X. 2011. Mathematical analyses of casino rebate systems for VIP gambling. International Gambling Studies, 11, 93-106.

HALL, P. 1997. The bootstrap and Edgeworth expansion, Springer Verlag.

HENDLER, R. \& HENDLER, F. 2004. Revenue management in fabulous Las Vegas: Combining customer relationship management and revenue management to maximise profitability. Journal of Revenue and Pricing Management, 3, 73-79.

MILLS, R. G. J. \& PANTON, D. M. 1992. SCHEDULING OF CASINO SECURITY OFFICERS. OmegaInternational Journal of Management Science, 20, 183-191.

NEVADA GAMING COMMISSION AND STATE GAMING CONTROL BOARD 2005. Technical Standards for Gaming Devices and On-line Slot Systems.

PRICEWATERHOUSECOOPER. 2010. Playing to Win [Online]. http://www.pwc.com/gx/en/entertainment-media/pdf/pwc-playing-to-win.pdf.

Available: $\quad$ http://www.pwc.com/gx/en/entertainment-media/pdf/pwc-playing-towin.pdf.

SMALL, C. G. 2010. Expansions and asymptotics for statistics, CRC Press.

SUGDEN, S. J. 2010. Aspects of Keno Modelling. Journal of Gambling Business and Economics, 4, 31-52.

TSOUKIAS, A. 2008. From decision theory to decision aiding methodology. European Journal of Operational Research, 187, 138-161.

WERTHAMER, N. R. 2005. Optimal Betting in Casino Blackjack. International Gambling Studies, 5, 253-270.

WERTHAMER, N. R. 2008. Optimal Betting in Casino Blackjack III: Table-Hopping. International Gambling Studies, 8, 63-75. 